\title{
Fire Containment in Planar Graphs
}

\author{
Louis Esperet* Jan van den Heuvel ${ }^{\dagger}$ \\ Frédéric Maffray ${ }^{\ddagger} \quad$ Félix Sipma ${ }^{\S}$
}

\begin{abstract}
In a graph $G$, a fire starts at some vertex. At every time step, firefighters can protect up to $k$ vertices, and then the fire spreads to all unprotected neighbours. The $k$-surviving rate $\rho_{k}(G)$ of $G$ is the expectation of the proportion of vertices that can be saved from the fire, if the starting vertex of the fire is chosen uniformly at random. For a given class of graphs $\mathcal{G}$ we are interested in the minimum value $k$ such that for some constant $\epsilon>0$ and all $G \in \mathcal{G}, \rho_{k}(G) \geq \epsilon$ (i.e., such that linearly many vertices are expected to be saved in every graph from $\mathcal{G})$.

In this note, we prove that for planar graphs this minimum value is at most 4 , and that it is precisely 2 for triangle-free planar graphs.
\end{abstract}

Keywords: the Firefighter Problem; surviving rate; planar graphs.

\section{Introduction}

The Firefighter Problem in graphs was introduced by Hartnell in 1995 [6]. In a graph $G$, a fire starts at time 0 at some vertex $v$ of $G$. At every subsequent time step, the firefighters protect at most $k$ vertices from the fire (this protection is permanent), and then the fire spreads to all unprotected neighbours. This problem has been heavily studied over the past decade; we refer the reader to a survey of Finbow and MacGillivray [4] for a general overview, and to [2, 3, 7] for specific algorithmic and complexity results.

* CNRS, Laboratoire G-SCOP, Grenoble, France; louis.esperet@g-scop.fr.

$\dagger$ Department of Mathematics, London School of Economics, London, UK; jan@maths.1se.ac.uk.

$\ddagger$ CNRS, Laboratoire G-SCOP, Grenoble, France; frederic.maffray@g-scop.inpg.fr.

$\S$ Laboratoire G-SCOP, Grenoble, France; felix.sipma@ens-lyon.org.

This work was partially supported by ANR Project HEREDIA, under grant (ANR-10-JCJC-HEREDIA).

Part of the research performed during a visit of JvdH to G-SCOP, Grenoble, supported by CNRS. JvdH likes to thank the members of G-SCOP for their hospitality. 
We denote by $s n_{k}(G, v)$ the maximum number of vertices of $G$ that can be saved from the fire if it starts at $v$. In general, the parameter $s n_{k}(G, v)$ depends heavily on $v$. For instance, if $G$ is a star on $n$ vertices, $v$ is the centre, and $u$ is a leaf, then $s n_{1}(G, v)=1$ while $s n_{1}(G, u)=n-1$. A good indicator of the robustness of a graph against a random fire is the following parameter introduced by Cai and Wang in 2009 [1]. They define the $k$-surviving rate $\rho_{k}(G)$ of a graph $G$ on $n$ vertices as $\frac{1}{n^{2}} \sum_{v \in G} s n_{k}(G, v)$. In other words, $\rho_{k}(G)$ is the expectation of the proportion of vertices that can be saved from the fire if it starts randomly in $G$.

For a family $\mathcal{C}$ of graphs, by a slight abuse of notation we use $\rho_{k}(\mathcal{C})$ to denote the infimum of $\rho_{k}(G)$ over all graphs $G \in \mathcal{C}$ with at least two vertices. We also define the firefighter number $f f(\mathcal{C})$ of the family $\mathcal{C}$ as the minimum integer $k$ such that $\rho_{k}(\mathcal{C})>0$. If no such value $k$ exists, we set $f f(\mathcal{C})=+\infty$.

Let $\mathcal{P}, \mathcal{P}_{g}$, and $\mathcal{O P}$ denote, respectively, the set of planar graphs, the set of planar graphs with girth (size of a shortest cycle) at least $g$, and the set of outerplanar graphs. Cai and Wang [1] proved that $\rho_{1}(\mathcal{O P}) \geq 1 / 6$, and asked the following.

Question 1 [1, Problem 6.2]

What is the minimum $k$ such that $\rho_{k}(\mathcal{P})>\epsilon$ for some $\epsilon>0$ ?

Using the notation introduced above, this is equivalent to asking for the value of $f f(\mathcal{P})$. Such a constant is at least two, as shown by the complete bipartite (planar) graph $K_{2, n-2}$ : When there is only one firefighter, only two vertices can be saved wherever the fire starts, hence $\rho_{1}\left(K_{2, n-2}\right)=2 / n$.

Wang et al. 8 . recently proved that $\rho_{1}\left(\mathcal{P}_{9}\right) \geq 2 / 35$, and that if a graph $G$ is $d$-degenerate (and has at least two vertices), $\rho_{2 d-1}(G) \geq 2 /(5 d)$. This implies that the firefighter number of every proper minor-closed class of graphs is finite. Wang et al. also proved that $\rho_{5}(\mathcal{P}) \geq 2 / 15$, which implies that $2 \leq f f(\mathcal{P}) \leq 5$.

The main purpose of this note is to prove the following results.

\section{Theorem 2}

(1) For the class $\mathcal{P}$ of planar graphs, we have $2 \leq f f(\mathcal{P}) \leq 4$.

(2) For the class $\mathcal{P}_{4}$ of triangle-free planar graphs, we have ff $\left(\mathcal{P}_{4}\right)=2$.

The proofs of these results can be found in Sections 2 and 3 . For the planar case, we indeed prove a much stronger theorem: we show that if 4 firefighters are available at the first step, and 3 firefighters at each subsequent step, then the surviving rate of every planar graph is bounded by a positive constant.

The main idea of the two proofs is to partition the vertices of a graph $G$ into two carefully chosen sets $X$ and $Y$. If the fire starts at a vertex of $X$, we will show that it 
can be quickly contained (saving all vertices but a constant number). If the fire starts at a vertex of $Y$, we will do nothing and let everything burn. We could easily save a couple of vertices by protecting them, but this would only make the computation harder (and only improve the constants). Then we will show that $|Y| \leq c|X|$ for some constant $c>0$, concluding the proof.

We illustrate this technique by proving the easy result below. The proofs in Sections 2 and 3 are more involved and use the well-known discharging method for planar graphs. The novel aspect of our approach is that we use the discharging method not just to prove that a particular configuration must exist at least once, but that it must exist many times.

\section{Theorem 3}

Any planar graph $G$ with girth at least 5 and at least two vertices satisfies $\rho_{2}(G) \geq 1 / 22$.

Proof From Euler's formula, it is easy to deduce that planar graphs with girth at least 5 have average degree less than 10/3; while planar graphs with girth at least 6 have average degree less than 3.

Let $X_{2}$ and $Y_{4}$ be the set of vertices of $G$ of degree at most 2 and at least 4 , respectively. Let $X_{3}$ be the set of vertices of degree 3 with a neighbour of degree at most 3 , and let $Y_{3}$ be the set of vertices of degree 3 not in $X_{3}$. We use $x_{2}, x_{3}, y_{3}, y_{4}$ to denote the cardinality of the sets $X_{2}, X_{3}, Y_{3}, Y_{4}$, respectively. Let $n$ be the number of vertices in $G$.

If the fire starts at $v \in X_{2}$, we protect its two neighbours, saving $n-1$ vertices. Consider a vertex $v \in X_{3}$, and let $u$ be its neighbour of degree at most 3 . If the fire starts at $v$, we first protect its two neighbours distinct from $u$. The fire then reaches $u$, and we protect the two neighbours of $u$ distinct from $v$, saving $n-2$ vertices. If the fire starts at a vertex from $Y_{3}$ or $Y_{4}$, we do nothing.

Since for a fire that starts at a vertex from $X_{2} \cup X_{3}$ we can save at least $n-2$ vertices, we obtain for the 2-surviving rate

$$
\rho_{2}(G)=\frac{1}{n^{2}} \sum_{v \in G} s n_{2}(G, v) \geq \frac{1}{n^{2}} \cdot\left(x_{2}+x_{3}\right)(n-2)=\frac{n-2}{n} \cdot \frac{x_{2}+x_{3}}{x_{2}+x_{3}+y_{3}+y_{4}} .
$$

Consider the subgraph $H$ of $G$ induced by the edges with one end in $Y_{3}$ and the other in $Y_{4}$. This graph has at most $y_{3}+y_{4}$ vertices and precisely $3 y_{3}$ edges. Since $H$ is bipartite and $G$ has girth at least $5, H$ has girth at least 6 . Hence, its average degree is less than 3 and we have $6 y_{3} \leq 3\left(y_{3}+y_{4}\right)$, implying that $y_{3} \leq y_{4}$.

Since the average degree in $G$ is less than 10/3, $3 x_{3}+3 y_{3}+4 y_{4} \leq \frac{10}{3}\left(x_{2}+x_{3}+y_{3}+y_{4}\right)$. Using that $y_{3} \leq y_{4}$, this implies $y_{4} \leq 10 x_{2}+x_{3}$, and hence $y_{3}+y_{4} \leq 20 x_{2}+2 x_{3} \leq$ $20\left(x_{2}+x_{3}\right)$. As a consequence, we obtain, using (11):

$$
\rho_{2}(G) \geq \frac{n-2}{n} \cdot \frac{x_{2}+x_{3}}{x_{2}+x_{3}+y_{3}+y_{4}} \geq \frac{n-2}{n} \cdot \frac{1}{1+\frac{y_{3}+y_{4}}{x_{2}+x_{3}}} \geq \frac{n-2}{21 n} .
$$


If $n \geq 44$, we obtain $\rho_{2}(G) \geq 1 / 22$. Otherwise, if $G$ has only two vertices, the vertex distinct from the firestart can be saved, while if $G$ has $3 \leq n \leq 44$ vertices, at least $2 / 44=1 / 22$ of the vertices can be saved. So in all cases we have $\rho_{2}(G) \geq 1 / 22$.

Theorem 3 has the following immediate consequence.

Corollary 4

For the class $\mathcal{P}_{5}$ of planar graphs with girth at least 5 , we have $1 \leq f f\left(\mathcal{P}_{5}\right) \leq 2$.

\section{Planar graphs}

In this section we prove the following theorem.

\section{Theorem 5}

Assume 4 firefighters are given at the first step, and then 3 at each subsequent step. Then the firefighters have a strategy such that every planar graph has surviving rate at least 1/2712.

Proof We can assume that $G$ is a maximal planar graph (hence a planar triangulation), since adding edges to the graph can only make things more difficult for the firefighters. Hence, $G$ has minimum degree at least 3 . For $3 \leq d \leq 6$, let $X_{d}$ be the set of vertices $v$ of degree $d$ so that if the fire starts at $v$, the firefighters have a strategy that saves at least $|V(G)|-6$ vertices; the other vertices of degree $d$ form the set $Y_{d}$. For $d \geq 7, Y_{d}$ is the set of all vertices of degree $d$. We set $X=\bigcup_{3 \leq d \leq 6} X_{d}$ and $Y=\bigcup_{d \geq 3} Y_{d}$.

Note that every vertex $v$ of degree $3 \leq d \leq 4$ is in $X_{d}$, since placing the firefighters on $v$ 's neighbours saves all the vertices except $v$. Hence, $Y_{3}$ and $Y_{4}$ are both empty. Also observe that if a vertex $v$ of degree 5 has a neighbour $u$ of degree at most 6 , then $v$ is in $X_{5}$ : first place four firefighters on the neighbours of $v$ distinct from $u$, the fire then spreads to $u$. Since $G$ is maximal planar only three unprotected neighbours of $u$ remain, which can be protected by three firefighters in the next step.

We now make a small observation about the sets $X_{6}$ and $Y_{6}$. The length of a path is the number of edges on the path.

Observation 6 For every vertex $v \in Y_{6}$, there is a path of length at most 3 connecting $v$ and a vertex $u$ of degree distinct from 6 , and such that all the internal vertices on the path have degree precisely 6.

Assume this is not the case. Then the subgraph of $G$ induced by the vertices at distance at most 3 from $v$ is the induced subgraph of a hexagonal grid. In this case, Figure 1 depicts a strategy for the firefighters saving all the vertices except at most six (which contradicts the 
fact that $v \in Y_{6}$ ). The figure should be read as follows: the fire starts at the squared vertex labelled 0 , then the firefighters protect the circled vertices labelled 1 , the fire spreads to all the squared vertices labelled 1 , the firefighters protect the circled vertices labelled 2 , and so on.

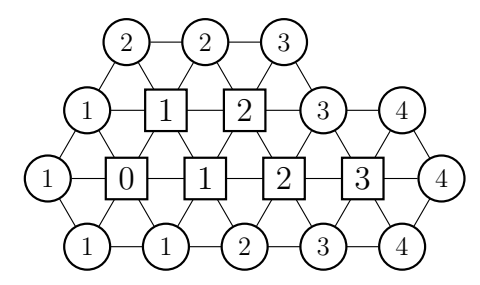

Figure 1: A strategy saving at least $n-6$ vertices if the neighbourhood of the firestart is a hexagonal grid.

For a planar graph with vertex set $V$, edge set $E$ and face set $F$, Euler's formula gives $|V|-|E|+|F|=2$. For simple maximal planar graphs, it is well known that this is equivalent to $\sum_{v \in V}(d(v)-6)=-12$. We interpret this by giving each vertex $v$ an initial charge $\sigma_{1}(v)=d(v)-6$. We redistribute this initial charge according to the following rules. Here the value of $\alpha$ will be determined later.

(R1) Each vertex of degree at least 7 gives a charge $1 / 4$ to each of its neighbours from $Y_{5}$.

(R2) For each vertex $v \in Y_{6}$ we choose one vertex $u$ with $d(u) \neq 6$ and $\operatorname{dist}(v, u) \leq 3$ (using Observation 60); this vertex $u$ gives a charge $\alpha$ to $v$.

The charge obtained after applying the rules (R1) and (R2) is denoted by $\sigma_{2}(v), v \in V$. Note that we have $\sum_{v \in V} \sigma_{2}(v)=\sum_{v \in V} \sigma_{1}(v)=-12$.

Observation 7 A vertex $v$ with $d(v) \geq 7$ has at most $\left\lfloor\frac{1}{2} d(v)\right\rfloor$ neighbours in $Y_{5}$.

This follows directly, since if $v$ has a neighbour $u$ in $Y_{5}$, then the two common neighbours of $u$ and $v$ must have degree at least 7 as well (otherwise $u$ is in $X_{5}$ ).

Claim 8 There is a constant $\alpha>0$ such that for every $v \in X$ we have $\sigma_{2}(v)>-3-93 \alpha$; while for every $v \in Y$ we have $\sigma_{2}(v) \geq \alpha$.

To prove the claim, we first estimate how often a vertex $v$ with $d(v) \neq 6$ can give a charge $\alpha$ according to (R2). As a very crude upper bound, this is at most the number of paths of length at most 3 , starting in $v$, and whose internal vertices all have degree exactly 6 . This number is clearly at most $d(v) \cdot(1+5+25)=31 d(v)$.

Each vertex $v$ of degree 3 gives at most $3 \times 31$ times a charge according to (R2). Since $\sigma_{1}(v)=-3$, this gives $\sigma_{2}(v) \geq-3-93 \alpha$. Similarly, for a vertex $v$ of degree 4 we have 
$\sigma_{2}(v) \geq-2-124 \alpha$; while for $v \in X_{5}$ we have $\sigma_{2}(v) \geq-1-155 \alpha$. Finally, for a vertex $v \in X_{6}$ we have $\sigma_{2}(v)=\sigma_{1}(v)=0$.

From rule (R2) it follows that if $v \in Y_{6}$, then $\sigma_{2}(v)=\sigma_{1}(v)+\alpha=\alpha$.

For a vertex $v$ with degree $d(v) \geq 7$, we can estimate, using the observations above,

$$
\sigma_{2}(v) \geq(d(v)-6)-\left\lfloor\frac{1}{2} d(v)\right\rfloor \cdot 1 / 4-31 d(v) \alpha .
$$

If $d(v)=7$, this gives $\sigma_{2}(v) \geq 1 / 4-217 \alpha$; and if $d(v) \geq 8$, we have the estimate $\sigma_{2}(v) \geq d(v) \cdot(7 / 8-31 \alpha)-6$.

We see that $\sigma_{2}(v) \geq \alpha>0$ for all $v \in Y$ if we can choose $\alpha>0$ such that $1 / 4-217 \alpha \geq \alpha$ and $d \cdot(7 / 8-31 \alpha)-6 \geq \alpha$ for all $d \geq 8$. It is easy to check that $\alpha=1 / 872$ will do the job. That value will also guarantee that $\sigma_{2}(v)>-3-93 \alpha$ for all $v \in X$, completing the proof of the claim.

The claim means that $-12=\sum_{v \in V} \sigma_{2}(v) \geq(-3-93 \alpha)|X|+\alpha|Y|$. This gives $|Y| \leq$ $(93+3 / \alpha)|X|=2709|X|$. So the surviving rate of a graph on $n=|X|+|Y|$ vertices with this strategy is at least

$$
\frac{n-6}{n} \cdot \frac{|X|}{|X|+|Y|}>\frac{n-6}{n} \cdot \frac{|X|}{2710|X|}=\frac{n-6}{2710 n} .
$$

So if $n \geq 10846$, the surviving rate is at least $1 / 2712$. On the other hand, if $2 \leq n<10846$, then we still can save at least $\min (4, n-1)$ vertices, hence the surviving rate in that case is still at least $4 / 10846>1 / 2712$.

Theorem 5 gives the upper bound on $f f(\mathcal{P})$ in Theorem $2(1)$ and the lower bound follows from the graph $K_{2, n}$ as considered earlier.

\section{$3 \quad$ Triangle-free planar graphs}

\section{Theorem 9}

Every triangle-free planar graph $G$ with at least two vertices satisfies $\rho_{2}(G) \geq 1 / 723636$.

Proof For a star $K_{1, n-1}, n \geq 2$, we have $\rho_{2}\left(K_{1, n-1}\right) \geq 1 / 2$, so we can assume $G$ is not a star.

Next we can assume that $G$ is edge-maximal with the property of being triangle-free and planar, since adding edges to the graph can only make things more difficult for the firefighters. As a consequence it is not difficult to see that $G$ is connected and, using the assumption that $G$ is not a star, in fact $G$ has no cut-vertex. This means the minimum degree is at least 2 . 
We assume some fixed embedding of $G$ in the plane. The embedding gives a circular order on the neighbours of a vertex. We use this order to talk about consecutive neighbours. Since $G$ is 2-connected, the degree of a face (the number of edges in a boundary walk of the face) is precisely the number of vertices incident with the face. We use $d(f)$ to denote the degree of a face.

For $2 \leq d \leq 4$, let $X_{d}$ be the set of vertices $v$ of degree $d$ such that if the fire starts at $v$, the two firefighters have a strategy that saves at least $|V(G)|-18$ vertices; the other vertices of degree $d$ form the set $Y_{d}$. For $d \geq 5, Y_{d}$ is the set of all vertices of degree $d$. We set $x_{d}=\left|X_{d}\right|$ and $y_{d}=\left|Y_{d}\right|, X=\bigcup_{2 \leq d \leq 4} X_{d}$, and $Y=\bigcup_{d \geq 2} Y_{d}$. Observe that every vertex $v$ of degree 2 is in $X_{2}$, since placing the firefighters on $v$ 's neighbours saves all the vertices except $v$. Hence, $Y_{2}$ is empty.

Two vertices $u$ and $v$ are 4-opposite if there is a face of degree 4 with boundary vertices $u, a, v, b$ in that order, where at least one of $a, b$ has degree 4 . Two vertices $u$ and $v$ are 4-adjacent if they are adjacent and the two faces incident with the edge $u v$ have degree 4 .

We now give some remarks about the set $X_{3}$.

Observation 10 Every vertex $v$ of degree 3 satisfying at least one of the following properties is in $X_{3}$ :

$3.1 v$ is adjacent to a vertex of degree at most 3;

$3.2 v$ is adjacent to a vertex of degree 4 having another neighbour of degree at most 3;

$3.3 v$ is 4-opposite to a vertex of degree at most 4 ;

$3.4 v$ is 4-opposite to a vertex of degree 5 having a neighbour of degree at most 3;

$3.5 v$ is adjacent to a vertex $w$ of degree 5 having three consecutive neighbours of degree at most 3 (including $v$ ), and such that the middle vertex from these three neighbours of $w$ is 4-adjacent to $w$;

$3.6 v$ is 4-adjacent to a vertex of degree 6 that is 4-adjacent to 6 vertices of degree at most 3.

To see this, consider Figures 2, 3, and 4, In each figure, the strategy of the firefighters is described in the same way we did for Figure 1 (for instance, the firestart $v$ is the squared vertex labelled 0 ). The degrees of the relevant vertices are indicated next to those vertices. Note that the exact order of the neighbours around the vertex of degree 4 and 5 in configurations 3.2 and 3.4, respectively, is not relevant and does not influence the strategy.

Using these observations, we now derive some useful properties of the vertices in $Y_{3}$. We need a few more definitions. An element is a vertex or a face. An element is contiguous with a vertex $v$, if it is either a face that is incident with $v$, or a vertex that is 4-opposite 

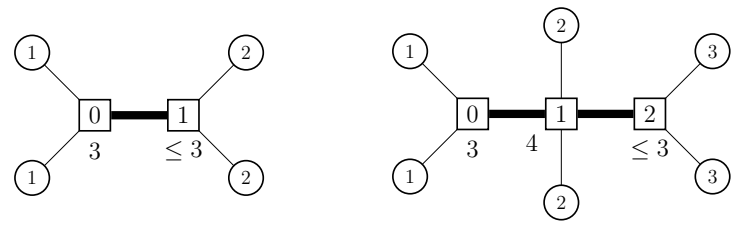

Figure 2: Configurations 3.1 (left) and 3.2 (right).
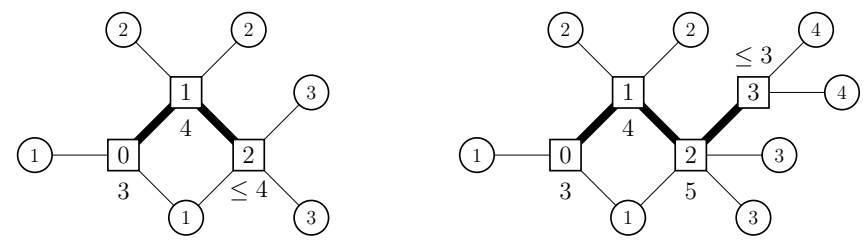

Figure 3: Configurations 3.3 (left) and 3.4 (right).
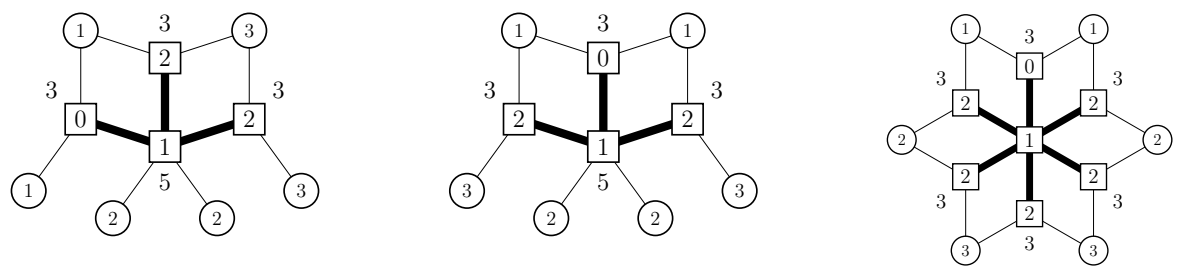

Figure 4: Configurations 3.5 (left and centre) and 3.6 (right).

or 4-adjacent to $v$. Two vertices $u$ and $v$ are 5-adjacent if they are adjacent and exactly one of the two faces incident with the edge $u v$ has degree 4.

Claim 11 For any $v \in Y_{3}$, at least two elements of degree at least 5 are contiguous with $v$. Moreover, if there are only two such elements, then $v$ is 5-adjacent to two more vertices of degree at least 5 and $v$ is incident with at least one face of degree at least 5 and at least one face of degree 4.

By 3.1 of Observation 10, all the neighbours of a vertex $v \in Y_{3}$ have degree at least 4 . If $v$ is adjacent to a vertex $u$ of degree 4 , then, by 3.3 of Observation 10, for each face $f$ incident with $u v$, either $d(f) \geq 5$ or there exists a vertex $w$ incident with $f$ such that $d(w) \geq 5$ and $v$ and $w$ are 4-opposite. So if $v$ is incident to zero or three faces of degree 4, then $v$ is contiguous with at least three elements of degree at least 5, and the claim holds.

If $v$ is incident to only one face of degree 4, then it is contiguous with two faces of degree at least 5. Moreover, by the above, either $v$ is 4-opposite (and so, contiguous) to a vertex of degree at least 5 , or it is 5 -adjacent to two vertices of degree at least 5 .

Finally, assume that $v$ is incident to precisely two faces of degree 4 . Then it is contiguous with a face $f$ of degree at least 5 . Let $u$ be the neighbour of $v$ such that $u v$ is not incident 
with $f$. By the above, if $d(u)=4$, then $v$ is 4-opposite to two vertices of degree at least 5 . So in this case it is contiguous with at least three elements of degree at least 5 . If $d(u) \geq 5$, then, since $u$ and $v$ are 4-adjacent, $v$ is contiguous with two elements of degree at least 5 . By the remark above, if no other element of degree at least 5 is contiguous with $v$, then the two neighbours of $v$ distinct from $u$ must have degree at least 5 , which completes the proof of the claim.

We denote by $Y_{3,2}$ the vertices in $Y_{3}$ that are only contiguous with two elements of degree at least 5 .

Claim 12 For any $v \in Y_{5}$, at most three vertices of $Y_{3}$ are contiguous with $v$. Moreover, if three vertices of $Y_{3}$ are contiguous with $v$, they consist of three non-consecutive neighbours of $v$, and all the faces incident with $v$ have degree 4.

We first observe that by 3.4 of Observation 10, a vertex $v \in Y_{5}$ cannot be both adjacent to a vertex $u \in Y_{3}$ and 4-opposite to a vertex $w \in Y_{3}$ (since otherwise $w$ would be in $X_{3}$ by definition). Assume that $v$ is 4-opposite to two consecutive vertices $x$ and $y$ of $Y_{3}$ (i.e., the faces that $v$ shares with $x$ and $y$ are consecutive with respect to $v$ ). By 3.1 and 3.2 of Observation 10, the common neighbour $z$ of $v, x, y$ cannot have degree less than 5 , so the two neighbours of $v$ distinct from $z$, but adjacent to $x$ or $y$, have degree 4 . The situation, together with a strategy for the firefighters in the case a fire starts at $x$, is depicted in Figure 5. This contradicts $x \in Y_{3}$. It follows that $v$ cannot be 4-opposite to three vertices

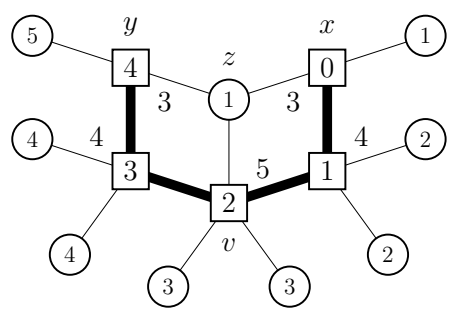

Figure 5: A vertex $v \in Y_{5}$ with two consecutive 4-opposite vertices $x, y \in Y_{3}$.

of $Y_{3}$. Assume now that $v$ is adjacent to some vertices of $Y_{3}$. By 3.5 of Observation 10, there cannot be three vertices of $Y_{3}$ that are 4-adjacent to $v$ and consecutive around $v$. Moreover, if three non-consecutive neighbours of $v$ are 4-adjacent to $v$, then all the faces incident with $v$ have degree 4 , which concludes the proof of Claim 12 .

We denote by $Y_{5,3}$ the vertices in $Y_{5}$ that are 4-adjacent to three vertices of $Y_{3}$. By the previous claim, we see that all the faces incident with a vertex of $Y_{5,3}$ have degree 4 , but a vertex of $Y_{3}$ cannot be 4-opposite to a vertex of $Y_{5,3}$.

Claim 13 Every vertex in $Y_{3}$ is 4 -adjacent to at most one vertex of $Y_{5,3}$. 
Assume the claim is false, and some vertex $\operatorname{vin} Y_{3}$ is 4 -adjacent to two vertices of $Y_{5,3}$. By Claim 12, only four cases need to be considered. In each of the cases, there is a strategy for the firefighters for a fire that starts at one of the vertices from $Y_{3}$ and saves at least $|V(G)|-7$ vertices; see Figure 6, But this contradicts the definition of a vertex in $Y_{3}$.
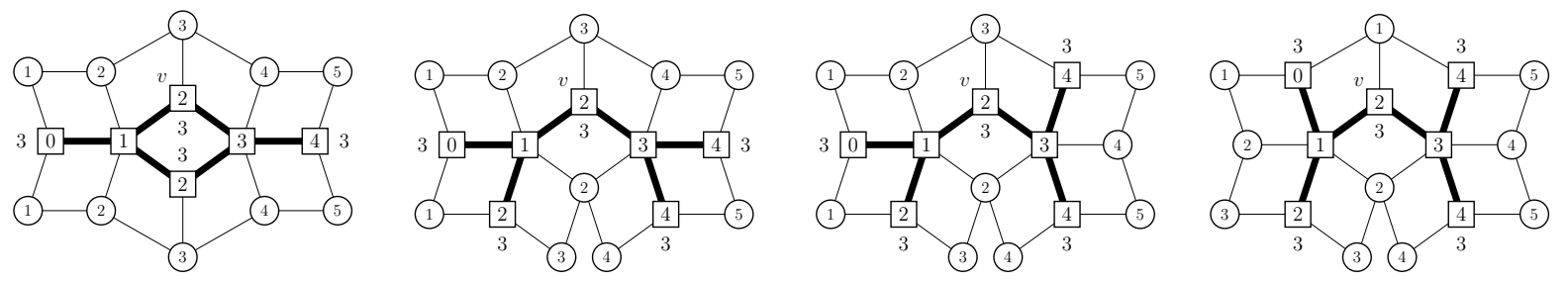

Figure 6: A vertex $v \in Y_{3}$ with two neighbours in $Y_{5,3}$.

Claim 14 If $v \in Y_{6}$, then at most six vertices of $Y_{3}$ are contiguous with or 5-adjacent to $v$. Moreover, if there are six such vertices, $v$ is 5-adjacent to at least two of them.

By 3.1 of Observation 10, if $v$ is 4-opposite to some vertex $u \in Y_{3}$, then the (at least two) common neighbours of $v$ and $u$ have degree at least 4, so they are not in $Y_{3}$. Assume that six or more vertices of $Y_{3}$ are contiguous with or 5-adjacent to $v$. By the remark above, this can only happen if either $v$ is 4-opposite to six vertices of $Y_{3}$, which contradicts 3.2 of Observation 10, or $v$ is 4 -adjacent or 5-adjacent to six vertices of $Y_{3}$. In the latter case, by 3.6 of Observation 10, $v$ is not allowed to be 4-adjacent to six vertices of $Y_{3}$, so $v$ must be incident with a face of degree at least 5 . Since not all faces can have degree at least 5 (since then $v$ cannot be 4 -adjacent or 5 -adjacent to any vertex), $v$ is 5 -adjacent to at least two vertices of $Y_{3}$.

The argument above involving 3.1 has the following consequence in general.

Claim 15 If $v \in Y_{d}$ with $d \geq 7$, at most $d$ vertices of $Y_{3}$ are contiguous with or 5-adjacent to $v$.

We finish this part with one observation regarding vertices in $Y_{4}$.

Observation 16 For every vertex $v \in Y_{4}$, there is a path of length at most 7 connecting $v$ and a vertex $u$ such that either $d(u) \neq 4$, or a face incident with $u$ and its neighbour on the path has degree at least 5. Moreover, all the internal vertices on the path and the faces incident with $v$ or with two internal vertices have degree precisely 4. 


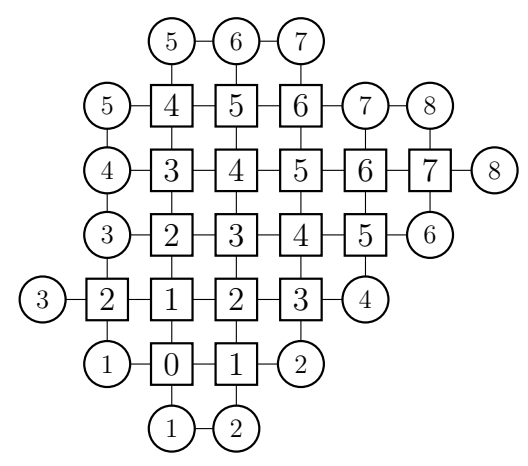

Figure 7: A strategy saving at least $n-18$ vertices if the neighbourhood of the firestart is a rectangular grid.

Assume this is not the case. Then the subgraph of $G$ induced by the vertices at distance at most 7 from $v$ is the induced subgraph of a rectangular grid. In this case Fogarty [5] gave a strategy saving all the vertices except at most 18 when the fire starts at $v$ (see Figure 7). This contradicts the fact that $v \in Y_{4}$.

We continue as in the proof of Theorem 5. We assign a charge $\nu_{1}(v)=d(v)-4$ to each vertex $v \in V$, and a charge $\nu_{1}(f)=d(f)-4$ to each face $f \in F$. Euler's formula gives $\sum_{v \in V} \nu_{1}(v)+\sum_{f \in F} \nu_{1}(f)=-8$. We redistribute this initial charge according to the following rules. Here the values of $\alpha$ and $\beta$ will be determined later.

(S1) A vertex in $Y_{5,3}$ gives a charge of $1 / 3-\beta$ to each of its three neighbours from $Y_{3}$.

(S2) A vertex of degree at least 5 not in $Y_{5,3}$ gives a charge of $2 / 5-\beta$ to each vertex in $Y_{3}$ it is contiguous with.

(S3) A vertex of degree at least 5 gives a charge of $1 / 10-\beta$ to each vertex in $Y_{3}$ it is 5 -adjacent to.

(S4) Each face of degree at least 5 gives a charge of $1 / 2-\beta$ to each vertex in $Y_{3}$ it is incident with.

(S5) For each vertex $v \in Y_{4}$ we choose a vertex $u$ with $\operatorname{dist}(v, u) \leq 7$ according to Observation 16, such that $\operatorname{dist}(v, u)$ is minimal. If $d(u) \neq 4$, this vertex $u$ gives a charge $\alpha$ to $v$. Otherwise, $u$ is incident to a face of degree at least 5 , which is at distance at most 6 from $v$; in that case this face gives a charge $\alpha$ to $v$.

The charge obtained after applying rules (S1)-(S4) is denoted by $\nu_{2}(x), x \in V \cup F$. Note that we have $\sum_{v \in V} \nu_{2}(v)+\sum_{f \in F} \nu_{2}(f)=\sum_{v \in V} \nu_{1}(v)+\sum_{f \in F} \nu_{1}(f)=-8$.

Claim 17 Let $\alpha=1 / 360720$ and $\beta=2186 \alpha$. Then for every $v \in X$ we have $\nu_{2}(v) \geq$ $-2-\beta$; for every $v \in Y$ we have $\nu_{2}(v) \geq \alpha$; and for every face $f, \nu_{2}(f) \geq 0$. 
To prove the claim, we first estimate how often a vertex $v$ with $d(v) \neq 4$ can give a charge $\alpha$ according to (S5). As a very crude upper bound, this is at most the number of paths of length at most 7 , starting in $v$, and whose internal vertices all have degree exactly 4 . This number is clearly at most $d(v) \cdot\left(1+3+3^{2}+\cdots+3^{6}\right)=1093 d(v)$. Hence, each vertex of degree $d$ gives at most $1093 d \alpha=\frac{1}{2} d \beta$ according to (S5).

It follows that a vertex $v$ of degree 2 satisfies $\nu_{2}(v) \geq \nu_{1}(v)-\beta=-2-\beta$. Similarly, for a vertex $v \in X_{3}$ we have $\nu_{2}(v) \geq-1-\frac{3}{2} \beta \geq-2-\beta$, since $\beta \leq 2$. And for $v \in X_{4}$ we have $\nu_{2}(v)=\nu_{1}(v)=0$.

Let $v \in Y_{3}$. Suppose first that $v$ is contiguous with three elements of degree at least 5 . By Claim 13, at most one of them is in $Y_{5,3}$, so $v$ receives at least $1 / 3+2 \times 2 / 5-3 \beta=$ $17 / 15-3 \beta$ by rules (S1), and (S2). Otherwise, by Claim 11, $v$ is only contiguous with two elements of degree at least 5, i.e., $v \in Y_{3,2}$. In this case we know that $v$ is incident to a face $f$ of degree at least 5 and 5 -adjacent to two vertices of degree at least 5 . Hence $v$ receives at least $1 / 2+1 / 3-2 \beta$ from $f$ and the second element of degree at least five it is contiguous with (by rules (S4), and (S1) or (S2)), and at least $2 \times(1 / 10-\beta)$ by rule (S3). So, in both cases $v$ receives at least $31 / 30-4 \beta$. Since it gives at most $\frac{3}{2} \beta$ according to (S5), we obtain $\nu_{2}(v) \geq 1 / 30-\frac{11}{2} \beta$. Using that $\alpha=1 / 360720$ and $\beta=2186 \alpha$, this implies $\nu_{2}(v) \geq \alpha$.

From rule (S5) it follows that if $v \in Y_{4}$, then $\nu_{2}(v)=\nu_{1}(v)+\alpha=\alpha$.

Next consider a vertex $v \in Y_{5,3}$. This gives three times a charge $1 / 3-\beta$ according to (S1), no charge according to (S3) by Claim 12, and at most $5 \times \frac{1}{2} \beta$ according to (S5). Since $\nu_{1}(v)=1$, we find $\nu_{2}(v) \geq 1-3 \times(1 / 3-\beta)-\frac{5}{2} \beta=\frac{1}{2} \beta$.

Consider now a vertex $v \in Y_{5} \backslash Y_{5,3}$. By Claim 12, $v$ is contiguous with at most two vertices from $Y_{3}$. Recall that by 3.4 of Observation 10, $v$ cannot be 4 -opposite to a vertex of $Y_{3}$ and adjacent to a vertex of $Y_{3}$. If $v$ is not contiguous with any vertex of degree at least 5 , it gives at most $5 \times(1 / 10-\beta)$ according to (S3). If $v$ is contiguous with one vertex of $Y_{3}$, then it gives $2 / 5-\beta$ according to (S2) and at most $4 \times(1 / 10-\beta)$ according to (S3). Suppose now that $v$ is contiguous with exactly two vertices from $Y_{3}$, say $u$ and $w$. If one of them in 4-opposite to $v$, then $v$ gives $2 \times(2 / 5-\beta)$ only. Otherwise, $v$ is 4 -adjacent with $u$ and $w$. If $v$ is 5 -adjacent to its three other neighbours, then we end up with configuration 3.5 of Observation 10, a contradiction. Hence $v$ can only be 5 -adjacent to two more vertices, and it gives $2 \times(2 / 5-\beta)$ according to (S2) and at most $2 \times(1 / 10-\beta)$ according to (S3). In all cases, $v$ gives at most $1-4 \beta$. Since $v$ gives at most $5 \times \frac{1}{2} \beta$ according to (S5), we have $\nu_{2}(v) \geq 1-(1-4 \beta)-\frac{5}{2} \beta=\frac{3}{2} \beta$.

For a vertex $v \in Y_{6}$, we have by Claim 14 that it gives at most five times a charge according to (S2). Moreover, if it gives precisely $5 \times(2 / 5-\beta)$, then it is not 5 -adjacent to any vertex of $Y_{3}$. Otherwise, it gives at most $4 \times(2 / 5-\beta)+2 \times(1 / 10-\beta)$. Hence, $v$ gives at most $\max \{2-5 \beta, 9 / 5-6 \beta\}=2-5 \beta$. Finally, $v$ also gives at most $6 \times \frac{1}{2} \beta$ according to (S5). As $\nu_{1}(v)=2$, we obtain $\nu_{2}(v) \geq 2-(2-5 \beta)-3 \beta=2 \beta$. 
For a vertex $v$ with $d(v) \geq 7$, we can estimate, using Claim 15 and the observations above:

$$
\nu_{2}(v) \geq(d(v)-4)-d(v) \cdot(2 / 5-\beta)-d(v) \cdot \frac{1}{2} \beta=d(v) \cdot\left(3 / 5+\frac{1}{2} \beta\right) \geq \frac{7}{2} \beta .
$$

We now estimate how often a face $f$ can give a charge $\alpha$ according to (S5). This is at most the number of paths of length at most 6 , starting at a vertex $u$ of degree 4 incident with $f$, and whose internal vertices (and faces incident with them) all have degree exactly 4 . This number is at most $2 d(f) \cdot\left(1+3+3^{2}+\cdots 3^{5}\right)=728 d(f)$, since such a vertex $u$ has at most two neighbours such that the faces incident with those neighbours all have degree 4 . Using that $\beta=2186 \alpha$, this implies that each face of degree $d$ gives at most $728 d \alpha \leq \frac{2}{5} d \beta$ according to (S5).

By 3.1 of Observation [10, a face $f$ has at most $\left\lfloor\frac{1}{2} d(f)\right\rfloor$ vertices of $Y_{3}$ on its boundary. Hence, for a face $f$ of degree 5 , we have $\nu_{2}(f) \geq 1-2 \times(1 / 2-\beta)-5 \times \frac{2}{5} \beta=0$. For a face $f$ of degree at least 6 , we obtain

$$
\nu_{2}(f) \geq(d(f)-4)-\frac{1}{2} d(f) \cdot(1 / 2-\beta)-d(f) \cdot \frac{2}{5} \beta=d(f) \cdot\left(3 / 4+\frac{1}{10} \beta\right)-4 \geq \frac{3}{5} \beta .
$$

Finally, for a face $f$ of degree 4 , we have $\nu_{2}(f)=\nu_{1}(f)=0$.

Putting the inequalities together, we see that $\nu_{2}(v) \geq-2-\beta$ for all $v \in X ; \nu_{2}(v) \geq$ $\min \left\{\alpha, \frac{1}{2} \beta\right\}=\alpha$ for all $v \in Y$; and $\nu_{2}(f) \geq 0$ for any face $f$, completing the proof of the claim.

The claim means that $-8=\sum_{v \in V} \nu_{2}(v)+\sum_{f \in F} \nu_{2}(f) \geq(-2-\beta)|X|+\alpha|Y|$. This gives $|Y|<(2186+2 / \alpha)|X|=723626|X|$. So the surviving rate of a graph on $n=|X|+|Y|$ vertices with this strategy is at least

$$
\frac{n-18}{n} \cdot \frac{|X|}{|X|+|Y|}>\frac{n-18}{n} \cdot \frac{|X|}{723627|X|}=\frac{n-18}{723627 n}
$$

So if $n \geq 1447272$, the surviving rate is at least $1 / 723636$. On the other hand, if $2 \leq n<$ 1447272 , then we still can save at least $\min \{2, n-1\}$ vertices, hence the surviving rate in that case is still at least $2 / 1447272=1 / 723636$.

Again, we have made no attempts to optimise the constants, in order to concentrate on making the exposition as clear as possible. For instance, the estimates for the number of times a vertex or a face gives a charge $\alpha$ according to rules (S5) can be improved significantly with a more careful analysis.

Theorem 9 together with the graph $K_{2, n}$ imply Theorem 2 (2). 


\section{Conclusion}

Regarding the firefighter number of planar graphs, we conjecture the following.

\section{Conjecture 18}

For the class $\mathcal{P}$ of planar graphs, we have $f f(\mathcal{P})=2$.

We believe that the proof of Theorem 5 can be modified to prove that for some $\epsilon>0$, every planar graph $G$ satisfies $\rho_{3}(G) \geq \epsilon$, which would give $f f\left(\mathcal{P}_{5}\right) \leq 3$. The only difference with our setting is that only three firefighters are available at the first round (instead of four 1 ). The main consequence is that not only $Y_{4}$ is not empty, but also the structure of $Y_{5}$ is more complicated. However, the argument concerning $Y_{6}$ will run smoothly using a strategy of Fogarty [5] in hexagonal grids.

Nevertheless, we feel the proof technique is too local to lead to a proof of $f f(\mathcal{P})=2$. For instance, the fact that only a constant number of steps are considered does not allow to design a good strategy (saving a linear number of vertices in average) with two firefighters in a very large hexagonal grid.

Wang et al. [8] proved that $f f\left(\mathcal{P}_{9}\right)=1$. The ideas of the proof of Theorem 9] can be adapted to prove that $f f\left(\mathcal{P}_{8}\right)=1$, since it is not hard to show that a worst case scenario in this case is if locally, every face close to the firestart has size 8, and around the boundary of these faces the vertices alternatingly have degree 2 and 4. (In other words, locally the graph looks like a subdivided rectangular grid.) In this case, a strategy similar to the strategy of Fogarty described in Section 3 will save at least $n-63$ vertices. We omit details here, since we believe that such a result would still be far from optimal. Indeed, we conjecture the following.

\section{Conjecture 19}

For the class $\mathcal{P}_{5}$ of planar graphs of girth at least 5 , we have $f f\left(\mathcal{P}_{5}\right)=1$.

We finish with a remark on the connection between separators and firefighters. For some constant $\epsilon>0$, an $\epsilon$-separator $S$ in a connected graph $G$ is a set of vertices whose removal yields at least two components of size at least $\epsilon|V(G)|$. If for $\epsilon|V(G)|$ vertices $v$ of $G$, there is an $\epsilon$-separator $S_{v}$ whose cardinality is at most the distance between $v$ and $S_{v}$, then $\rho_{1}(G)>\epsilon^{2}$ : by the time a fire starting at $v$ reaches $S_{v}$, the single firefighter can protect all the vertices of $S_{v}$, so a (linear-sized) component of $G \backslash S_{v}$ not containing $v$ will be saved.

\footnotetext{
${ }^{1}$ It was pointed out by one of the referees that adding one firefighter at the first round can have significant implications in terms of complexity. In the usual setting (only one firefighter at each round), deciding whether a given number of vertices of a rooted subcubic tree can be saved if the fire starts at the root is NP-complete [3]. If a second firefighter is available at the first round only, the problem becomes trivially polynomial.
} 


\section{Acknowledgement}

The authors thank the anonymous referees for comments and suggestions. We also would like to thank Jiangxu Kong from Zhejiang Normal University, China for pointing out an error in an earlier version of the proof of Theorem 9 .

\section{References}

[1] L. Cai and W. Wang, The surviving rate of a graph for the firefighter problem. SIAM J. Discrete Math. 23 (2009), 1814-1826.

[2] C. Duffy, A Collection of Algorithmic and Complexity Results for Variants of the Firefighter Problem. MSc Thesis, Department of Mathematics and Statistics, University of Victoria, 2011.

[3] S. Finbow, A.D. King, G. MacGillivray, and R. Rizzi, The firefighter problem for graphs of maximum degree three. Discrete Math. 307 (2007), 2094-2105.

[4] S. Finbow and G. MacGillivray, The Firefighter Problem: A survey of results, directions and questions. Australas. J. Combin. 43 (2009), 57-78.

[5] P. Fogarty, Catching the Fire on Grids. MSc Thesis, Department of Mathematics, University of Vermont, 2003.

[6] B. Hartnell, Firefighter! An Application of Domination. Presentation, 25th Manitoba Conference on Combinatorial Mathematics and Combinatorial Computing, University of Manitoba in Winnipeg, Canada, 1995.

[7] A.D. King and G. MacGillivray, The firefighter problem for cubic graphs. Discrete Math. 310 (2010), 614-621.

[8] W. Wang, S. Finbow, and P. Wang, The surviving rate of an infected network. Theoret. Comput. Sci. 411 (2010), 3651-3660. 